\title{
Conserved genes in a path from commensalism to pathogenicity: comparative phylogenetic profiles of Staphylococcus epidermidis RP62A and ATCCI 2228
}

\author{
Wu Wei ${ }^{\dagger 1,2}$, ZhiWei Cao ${ }^{\dagger 2}$, Yu-Li Zhu ${ }^{3}$, XiaoJing Wang1,2, GuoHui Ding1, \\ Hao Xu${ }^{2}$, PeiLin Jia1,2, Di Qu*3, Antoine Danchin*4 and YiXue Li*1,2
}

Address: ${ }^{1}$ Bioinformatics Center, Shanghai Institutes for Biological Sciences, Chinese Academy of Sciences; Graduate School of the Chinese Academy of Sciences, 320 YueYang Road, Shanghai, China, 2Shanghai Center for Bioinformation Technology, 100 Qinzhou Road, Shanghai, China, ${ }^{3}$ Department of Medical Molecular Virology, Institute of Medical microbiology, Shanghai Medical School of Fudan University, 138 YiXue Yuan Road, Shanghai, China and ${ }^{4}$ Genetics of Bacterial Genomes/URA 2171 CNRS, Institut Pasteur, 28 rue du Docteur Roux, 75724 Paris Cedex 15, France

Email: Wu Wei - wwei@sibs.ac.cn; ZhiWei Cao - zwcao@scbit.org; Yu-Li Zhu - yulizhu@fudanyueda.com; XiaoJing Wang -xjwang@scbit.org; GuoHui Ding - gwding@sibs.ac.cn; Hao Xu - haoxu@scbit.org; PeiLin Jia - pljia@sibs.ac.cn; Di Qu* - dqu@shmu.edu.cn;

Antoine Danchin* - antoine.danchin@normalesup.org; YiXue Li* - yxli@scbit.org

* Corresponding authors †Equal contributors

Published: 10 May 2006

BMC Genomics 2006, 7:1 12 doi:10.1186/1471-2164-7-1/2
Received: 0I March 2006

Accepted: 10 May 2006

This article is available from: http://www.biomedcentral.com//47/-2/64/7//I2

(c) 2006 Wei et al; licensee BioMed Central Ltd.

This is an Open Access article distributed under the terms of the Creative Commons Attribution License (http://creativecommons.org/licenses/by/2.0), which permits unrestricted use, distribution, and reproduction in any medium, provided the original work is properly cited.

\begin{abstract}
Background: Staphylococcus epidermidis, long regarded as an innocuous commensal bacterium of the human skin, is the most frequent cause of nosocomial infections associated with implanted medical devices. This conditional pathogen provides a model of choice to study genome landmarks correlated with the transition between commensalism and pathogenicity. Traditional investigations stress differences in gene content. We focused on conserved genes that have accumulated small mutation differences during the transition.

Results: A comparison of strain ATCCI2228, a non-biofilm forming, non-infection associated strain and strain RP62A, a methicillin-resistant biofilm clinical isolate, revealed consistent variation, mostly single-nucleotide polymorphisms (SNPs), in orthologous genes in addition to the previously investigated global changes in gene clusters. This polymorphism, scattered throughout the genome, may reveal genes that contribute to adaptation of the bacteria to different environmental stimuli, allowing them to shift from commensalism to pathogenicity. SNPs were detected in 93 I pairs of orthologs with identical gene length, accounting for approximately $45 \%$ of the total pairs of orthologs. Assuming that non-synonymous mutations would mark recent evolution, and hence be associated to the onset of the pathogenic process, analysis of ratios of non-synonymous SNPs vs synonymous SNPs suggested hypotheses about possible pathogenicity determinants. The $N / S$ ratios for virulence factors and surface proteins differed significantly from that of average SNPs. Of those gene pairs, 40 showed a disproportionate distribution of $d \mathrm{~N}$ vs $\mathrm{dS}$. Among those, the presence of the gene encoding methionine sulfoxide reductase suggested a possible involvement of reactive oxygen species. This led us to uncover that the infection associated strain was significantly more resistant to hydrogen peroxide and paraquat than the environmental strain. Some 16 genes of the list were of unknown function. We could suggest however that they were likely to belong to surface proteins or considered in priority as important for pathogenicity.
\end{abstract}

Conclusion: Our study proposed a novel approach to identify genes involved in pathogenic processes and provided some insight about the molecular mechanisms leading a commensal inhabitant to become an invasive pathogen. 


\section{Background}

Emerging diseases have been a matter of great concern recently: the sudden appearance of SARS and the recent spread of bird's flu raise the question of how microbes evolve into pathogens to human not only an important but also an urgent question to answer. Most microbes have a benign symbiotic relationship with humans and only cause infections to healthy individuals under limited conditions. Staphylococcus epidermidis is such an opportunistic pathogen which is a common member of the normal flora of our skin and mucous membranes. But on certain occasions, the presence of $S$. epidermidis as a contaminant of medical devices, or breach of the skin by trauma or inoculation needles, makes it emerge as a causative agent of infections [1]. As biomedical devices are increasingly used in medical practice, a major complication due to $S$. epidermidis infection when using these devices is affecting several millions of patients worldwide each year [2].

The pathogenic process of foreign-body-associated infections with $S$. epidermidis is characterized by the ability of this species to colonize polymer surfaces by the formation of multilayered cell clusters, which are enveloped and protected by an amorphous slimy material to form a biofilm [3-5]. Most genetic and biochemical evidence has shown that polysaccharide intercellular adhesion (PIA) production [6], mediated by the icaABCD operon [7-9], is crucial in biofilm fomation. S. epidermidis does not produce components that are easily recognized as virulence factors, such as toxins or aggressive degradative exoenzymes [10]. Genetic manipulation of S. epidermidis has been difficult so far, limiting efforts that would elucidate the molecular basic of its pathogenicity. The analysis of bacterial genome sequences provides us with an alternative way to investigate some of the constraints that might pave the way from commensalism to pathogenicity in this species.

Staphylococcus epidermidis is one of the most commonly isolated bacterial pathogens in hospitals because of its large numbers and ubiquitous distribution. Environmental S. epidermidis strains differ significantly in their invasive and ability to form biofilm. Two complete genomes of $S$. epidermidis strains have been sequenced, one is ATCC 12228 , a non-biofilm forming/non-infection associated strain, and another one is RP62A (also called ATCC35984), an infectious and biofilm forming strain $[11,12]$. Both strains are highly similar in genome sequence, and we tried to see whether some particular features might be correlated with the surviving ability of the organisms in certain environment, allowing us to uncover processes that might be important for the transition between a commensal life style, and a pathogenic behavior. In previous comparative genomics studies, the most obvious differences between these genome pairs revealed the loss or gain of large DNA segments [13]. Further comparative studies of $S$. epidermidis with other staphylococcal species indicated that the majority of the genes unique to a given strain or species is also related to the presence or absence of prophages and genomic islands. In the absence of other criteria to define virulence, some of these genes were proposed to be the main causes of pathogenicity and virulence [12]. However, acquisition of those genes has repeatedly been reported to be insufficient to trigger a pathogenic response [14]. For example, it has been discovered that the ATCC12228 and RP62A strains share most of the possible pathogenic factors. As a case in point, the cap operon encoding the polyglutamate capsule and being recognized as a major virulence factor in Bacillus anthracis, has been found to be integrated into the genomes of both S. epidermidis RP62A and ATCC12228, while the latter is a non-infectious strain $[12,15]$. In addition to gross differences displayed as genomic islands and unique genes, Single Nucleotide Polymorphisms (SNPs) have also been found to widely exist in the chromosomes of the two strains, especially in some genes with cell envelope functions [12]. Therefore, pathogenicity appears to be a complex phenomenon which could be accounted for not only by large genetic differences, but also by small variations in gene contents. This prompted us to perform a detailed SNPs comparative analysis between $S$. epidermidis ATCC12228 and RP62A, complementing the previous approaches used to differentiate pathogenic strains from non-pathogenic ones [16-18]. Assuming that pathogens have evolved recently [19], the amount of small genetic variations that would be accumulated in the genome sequences might not have had time to undergo purifying selection, thus revealing some of the genes that are important for pathogenic processes. Analyzing those variations, mostly SNPs, we expected to find genes bearing landmarks of recent evolution, and which may contribute to the pathogenesis of the bacterium. In this paper, we focused on the systematic SNP analysis between a pair of infectious and non-infectious strains of $S$. epidermidis, in particular by comparing synonymous and non-synonymous mutations in orthologous gene pairs. The genes displaying higher $\mathrm{dN} / \mathrm{dS}$ rate, including genes of unknown function, were analyzed in relation with function possibly associated to pathogenicity.

\section{Results \\ Small variations between the genomes of two S. epidermidis strains}

The S. epidermidis RP62A and ATCC12228 chromosomes are 2,616,530 bp and 2,499,279 bp long, respectively $[11,12]$. Beside large segments coding for genes present in one genome and absent in the other, many small scale genetic variations which affected an individual gene or a small numbers of genes (< 10 CDSs) were dispersed throughout nearly all of both chromosomes, revealing 
Table I: Comparison of the CDSs of S. epidermidis ATCC I 2228 and S. epidermidis RP62A

\begin{tabular}{lcc}
\hline & S. epidermidis ATCC12228a & S. epidermidis RP62A \\
\hline Total CDS number & 2419 & 2494 \\
CDSs categorized by sequence variations & 2094 & 2103 \\
All homologs & 2053 & 2053 \\
$\quad$ Total orthologs & 859 & 859 \\
$\quad$ Orthologs with identical DNA sequences & 931 & 931 \\
$\quad$ Orthologs with SNPs and the same length & 263 & 263 \\
$\quad$ Orthologs with insertions/deletions & 41 & 50 \\
Paralogs & 325 & 391 \\
Unique compared to the other S. epidermidisc & 288 & 315 \\
$\quad$ Unique compared to all staphylococcal & & \\
\hline
\end{tabular}

a Genomic data of S. epidermidis ATCCI2228 (accession no. AEOI5934) and S. epidermidis RP62A (accession no. CP000029) are according to GeneBank.

b These CDSs were used in analyzing the variation of single-nucleotide polymorphisms (SNPs) between two S. epidermidis genome.

c These CDSs were specific to each other.

extensive divergence between both species. The comparison of the insertions and deletions (indels) differentiating the two genome sequences showed that such events generally involved small-scale variations (see Additional file 1). In addition to indels, a total of 10,297 SNPs were found in the genome of $S$. epidermidis ATCC12228 when compared to that of strain RP62A [12]. These small-scale variations, mostly SNPs, might contribute to the different phenotypes of the two strains. We therefore focused on the SNPs of the orthologous genes to explore how they could contribute to adaptation of the bacteria to various environmental stimuli allowing them to shift from commensalism to pathogenicity.

The S. epidermidis RP62A and ATCC12228 chromosomes contain 2,494 and 2,419 predicted protein-coding sequences (CDSs), respectively $[11,12]$. Of the 2,419 predicted genes encoded by the ATCC12228 chromosome, $2,053(85 \%)$ have an ortholog in RP62A (Table 1). Most of those genes are almost identical in sequence between both organisms. SNPs were detected in 931 pairs of orthologous genes with identical gene length, accounting for approximately $45 \%$ of the orthologous genes set (see Additional file 2). Among those orthologs with SNPs and of identical length, 118 pairs were identical to each other at the amino acid level, while the other 813 pairs (87\%) displayed some changes in their amino acid sequence. In addition, more complex insertion/deletion events and related variations were observed in 263 pairs of orthologs.

\section{Pathogenicity-related genes and highly expressed proteins genes evolve differently}

Pathogenicity in warm blooded vertebrates is expected to have arisen recently (at the living organisms evolution time scale). Mutations occurring during that period should have started more or less randomly, while selection pressure would have retained only some of them. This prompted us to measure the contribution of synonymous mutations (presumably not changing the existing fitness of the proteins) as compared to non-synonymous mutations. In order to assess the effect of non-synonymous mutations upon the intraspecies differentiation of the two S. epidermidis strains, we calculated the ratios of total non-synonymous SNPs $(\mathrm{N})$ vs total synonymous SNPs (S) of all genes, with some emphasis on virulence factors, surface proteins [12] and translation, ribosomal structure and biogenesis-related proteins (see Additional file 3 and file 4). This comparison would be a first proof

Table 2: Comparison of SNP frequency and distribution into different functional groups

\begin{tabular}{|c|c|c|c|c|c|c|c|c|}
\hline Groups $^{\mathrm{a}}$ & No.CDS & $n+s$ & $n^{b}$ & $\mathrm{~s}$ & $\mathrm{n} / \mathrm{s}$ & $P_{1}{ }^{c}$ & $P_{\| 1}$ & $P_{\mathrm{III}}$ \\
\hline I & 11 & 112 & 47 & 65 & 0.723 & & & \\
\hline II & 6 & 84 & 34 & 50 & 0.68 & 0.834 & & \\
\hline III & 48 & 400 & 99 & 301 & 0.329 & $0.000 *$ & $0.003^{*}$ & \\
\hline Total & 931 & 7322 & 2151 & 5171 & 0.416 & $0.003 * d$ & $0.025 * d$ & $0.047^{* d}$ \\
\hline
\end{tabular}

aGroups defined as (I) genes whose products are virulence factors and genomic islands in both S. epidermidis genome; (II) genes whose products are surface proteins; (III) genes whose products belong to translation, ribosomal structure and biogenesis catalog according to COG.

$\mathrm{b}(\mathrm{n})$ number of non-synonymous SNPs; (s) number of synonymous SNPs; $(\mathrm{n} / \mathrm{s})$ ratios of non-synonymous SNPs vs. synonymous SNPs.

$c P$-value from $\chi^{2}$ test for the difference in $\mathrm{n} / \mathrm{s}$ ratios in all the pairwise comparisons among the three functional groups $(*$ indicates $p<0.05)$.

$\mathrm{d} P$-value from $\chi^{2}$ test for the difference in $\mathrm{n} / \mathrm{s}$ ratios between the three functional groups and total without themselves $(*$ indicates $p<0.05)$. 
A

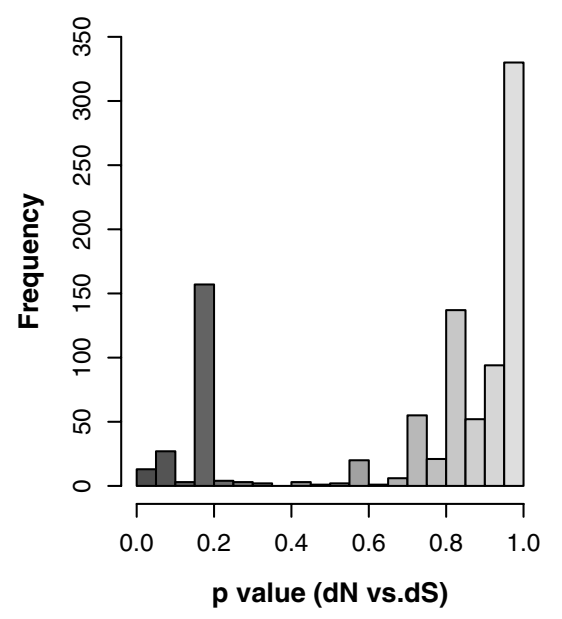

B

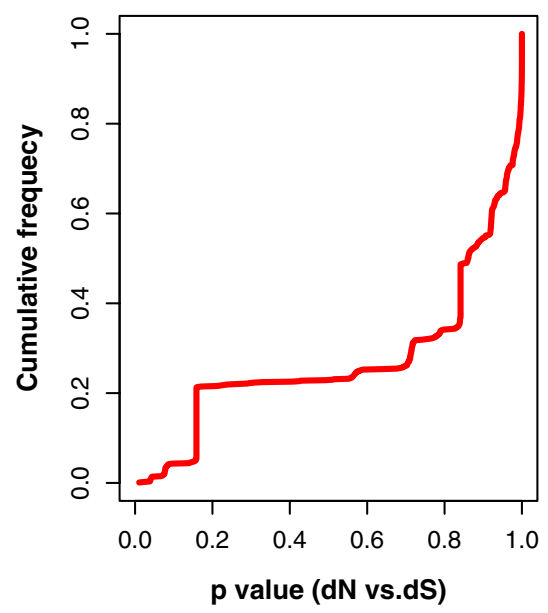

C

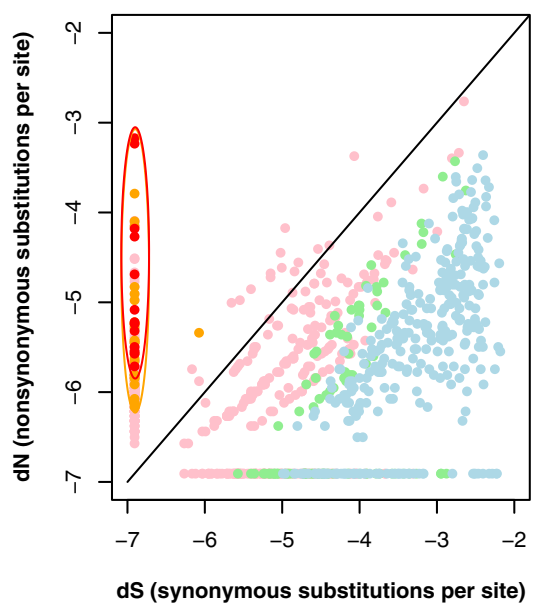

Figure I

Distribution of dN vs dS of SNPs pairs of two S. epidermidis strains. The distribution of dN vs dS mutations was represented according to the $p$-value calculated from the One-tail Z (or $t_{\infty}$ ) test. Figure IA represents the numbers of SNPs pairs falling into the different confidence level classes. Figure IB shows the cumulative probability of all SNPs pairs. Figure IC plots the distribution of $\mathrm{dN} / \mathrm{dS}$ of all SNPs pairs. All plots are color-coded according to different intervals: red for [0, 0.05$)$, orange for $[0.05,0.1)$, green for $(0.9,0.95]$, blue for $(0.95, I]$, pink for others.

of concept, while we were trying to uncover genes' illuminating or unexpected functions that would, in this way, suggest a participation in the evolution of pathogenicity. The N/S ratios for virulence factors and surface proteins differed significantly from that of all SNPs. In contrast, translation-related proteins also showed a significant bias when compared to total SNPs, but displayed mostly synonymous substitutions, indicative of a selective stabilization process leading to purifying selection(Table 2). This analysis shows that virulence factors and surface proteins evolved quickly, in parallel with the pathogenicity environment. That this is significant is emphasized by the observation that translation, ribosomal structure and biogenesis-related proteins, which are submitted to considerable structural and functional constraints and are highly expressed, evolved slowly (there is hardly any change in protein sequence, while the gene sequence has evolved) [20].

To identify groups of SNPs genes differing in their evolution pattern, the dS and dN of each SNPs pairs of the two S. epidermidis strains were calculated and the distribution of individual SNPs pairs were analyzed using the one-tail $\mathrm{Z}$ (or $t_{\infty}$ ) test (see Additional file 5). Among 931 pairs of orthologs with SNPs, 213 (23\%) pairs had a majority of non-synonymous substitutions $(\mathrm{dN}>\mathrm{dS})$, while the remaining $718(77 \%)$ pairs had a majority of synonymous mutations (Figure 1A and 1B). This suggested that the majority of orthologs with SNPs suffer considerable selection constraints, indicative of adaptation to similar environments for both strains. The others, in contrast, may contribute to the bacterial pathogenesis process during the establishment of infection. In those synonymous pairs, we therefore restricted our analysis to the confidence interval of $\mathrm{p}<0.1$ of $\mathrm{dS} / \mathrm{dN}$ to find what kinds of genes significantly suffered purifying selection pressures. As mentioned above, many genes of the translation machinery and energy production were found in that category. It is worth noticing that genes involved in the translation machinery, such as ribosomal proteins and tRNA methyltransferases, are expected to be expressed at a high level in many transcriptome studies [21].

\section{Genes potentially involved in pathogenicity}

A total of 40 genes showed a significantly disproportionate distribution of $\mathrm{dN}$ vs $\mathrm{dS}$ with p-value $<0.10$ (Figure $1 \mathrm{~A}$ and Table 3). In standard statistical analyses, $\mathrm{p}<0.05$ is the level traditionally considered significant; however, if we represented the relative amount of those genes, the plot distribution of $\mathrm{dN} / \mathrm{dS}$ (Figure 1C) shows a continuum that is worth exploring and may be relevant as providing putative signatures of adaptation to pathogenicity. Because the organisms we have chosen are extremely similar, leading to very few differences between the two genomes, the advantage of using a stringent statistical constraint for discarding false positives will put aside false negatives under conditions where we need to explore as many paths as possible. Indeed, from the figure we can see 
Table 3: Non-synonymous substitutions significant group $(p<0.10)$ of Orthologs with SNP Pairs potential involves in pathogenicity

\begin{tabular}{|c|c|c|c|c|c|c|c|}
\hline \multirow{2}{*}{$\begin{array}{l}\text { S. epidermidis } \\
\text { ATCCI } 2228\end{array}$} & \multirow{2}{*}{$\begin{array}{l}\text { S. epidermidis } \\
\text { RP62A }\end{array}$} & \multicolumn{2}{|l|}{ Function } & \multirow[t]{2}{*}{$\mathrm{N}$} & \multirow[t]{2}{*}{$S$} & \multirow[t]{2}{*}{$d N /(d S+d N)$} & \multirow[t]{2}{*}{$P$ value } \\
\hline & & Annotation from ATCCI 2228 & New annotation & & & & \\
\hline SEI029 & SERP09|8 & exonuclease SbcC & & 5 & 0 & 1.0000 & 0.0107 \\
\hline SE023I & SERP2349 & fosfomycin resistance protein fofB & & 4 & 0 & 1.0000 & 0.0228 \\
\hline SE0800 & SERP0689 & potD protein & & 3 & 0 & 1.0000 & 0.0381 \\
\hline SE0265 & SERP23 I 3 & hypothetical protein & Tranmembrane protein & 3 & 0 & 1.0000 & 0.0383 \\
\hline SEI546 & SERPI399 & D-3-phosphoglycerate dehydrogenase & & 3 & 0 & 1.0000 & 0.0392 \\
\hline SE0378 & SERP0259 & hypothetical protein & Tranmembrane protein & 3 & 0 & 1.0000 & 0.0398 \\
\hline SE2085 & SERP2099 & gluconokinase & & 3 & 0 & 1.0000 & 0.0401 \\
\hline SEI042 & SERP093I & peptide methionine sulfoxide reductase & & 3 & 0 & 1.0000 & 0.0405 \\
\hline SE0039 & SERP2495 & conserved hypothetical protein & & 3 & 0 & 1.0000 & 0.0406 \\
\hline SE0977 & SERP0866 & glycerol uptake facilitator & & 3 & 0 & 1.0000 & 0.0415 \\
\hline SEII70 & SERPI049 & probable ATP-dependent DNA helicase RecQ & & 3 & 0 & 1.0000 & 0.0425 \\
\hline SE0502 & SERP0385 & choline transport ATP-binding protein & & 3 & 0 & 1.0000 & 0.0427 \\
\hline SE0546 & SERP043I & conserved hypothetical protein & YvcD & 3 & 0 & 1.0000 & 0.0440 \\
\hline SEI 302 & SERPII 83 & deoxyribonuclease & & 2 & 0 & 1.0000 & 0.0668 \\
\hline SEI527 & SERPI 382 & conserved hypothetical protein & YheB (surface protein) & 2 & 0 & 1.0000 & 0.0707 \\
\hline SE2166 & SERP2I 77 & glycine betaine aldehyde dehydrogenase gbsA & & 2 & 0 & 1.0000 & 0.0719 \\
\hline SE2379 & SERP0037 & cystathionine gamma-synthase & & 2 & 0 & 1.0000 & 0.0743 \\
\hline SEI828 & SERPI836 & DNA topoisomerase III topB & & 2 & 0 & 1.0000 & 0.0743 \\
\hline SEI 824 & SERPI83I & 50 S ribosomal protein $\mathrm{L} 3$ & & 2 & 0 & 1.0000 & 0.0759 \\
\hline SE0309 & SERP0I86 & $30 \mathrm{~S}$ ribosomal protein $\mathrm{S} / 2$ & & 2 & 0 & 1.0000 & 0.0766 \\
\hline SE0463 & SERP0349 & putative deoxyribodipyrimidine photolyase & & 2 & 0 & 1.0000 & 0.0766 \\
\hline SE2067 & SERP2080 & glycine betaine aldehyde dehydrogenase gbsA & & 2 & 0 & 1.0000 & 0.0766 \\
\hline SE2215 & SERP2247 & arginine/oirnithine antiporter & & 2 & 0 & 1.0000 & 0.0766 \\
\hline SE0806 & SERP0695 & conserved hypothetical protein & YlaF & 2 & 0 & 1.0000 & 0.0768 \\
\hline SEI448 & SERPI335 & conserved hypothetical protein & YqgE (MultiDrug Efflux proteins) & 2 & 0 & 1.0000 & 0.0775 \\
\hline SE0847 & SERP0737 & hypothetical protein & phenol soluble modulin beta I & 2 & 0 & 1.0000 & 0.0777 \\
\hline SE0790 & SERP0679 & conserved hypothetical protein & YkyA (surface protein) & 2 & 0 & 1.0000 & 0.0779 \\
\hline SE2196 & SERP2207 & conserved hypothetical protein & & 2 & 0 & 1.0000 & 0.0781 \\
\hline SE2269 & SERPOI53 & dihydropteroate synthase chain $A$ synthetase & & 2 & 0 & 1.0000 & 0.0783 \\
\hline SE0744 & SERP0630 & 2-oxoglutarate decarboxylase & & 2 & 0 & 1.0000 & 0.0783 \\
\hline SE2003 & SERP20I6 & conserved hypothetical protein & & 2 & 0 & 1.0000 & 0.0783 \\
\hline SEI 247 & SERPII 26 & conserved hypothetical protein & YqfN & 2 & 0 & 1.0000 & 0.0787 \\
\hline SEI986 & SERPI998 & conserved hypothetical protein & In larger Bacterial clades & 2 & 0 & 1.0000 & 0.0800 \\
\hline SE0432 & SERP03I 7 & low-affinity inorganic phosphate transporter & & 2 & 0 & 1.0000 & 0.0808 \\
\hline SEI745 & SERPI754 & conserved hypothetical protein & YhfK & 2 & 0 & 1.0000 & 0.0827 \\
\hline SE0674 & SERP0564 & clpB protein & & 2 & 0 & 1.0000 & 0.0846 \\
\hline SE0773 & SERP0660 & conserved hypothetical protein & Cation transporter & 2 & 0 & 1.0000 & 0.0846 \\
\hline SEI608 & SERPI46I & beta-lactamase & & 2 & 0 & 1.0000 & 0.0846 \\
\hline SE2 128 & SERP2 140 & conserved hypothetical protein & YfmM & 2 & 0 & 1.0000 & 0.0874 \\
\hline SE0506 & SERP0389 & alcohol dehydrogenase & & 7 & I & 0.7451 & 0.0953 \\
\hline
\end{tabular}


that $213(23 \%)$ genes of the 931 SNP ortholog pairs are under positive selection $(\mathrm{dN} / \mathrm{dS}>1)$, consistent with the observation of other works [22] and strongly suggesting that most, if not all of the retained non-synonymous mutations are significant. Furthermore, the accumulation of mutations in some genes can be the result of several independent processes, with some submitted to positive selection for variation, while others would only be affected by drift, precluding, at this stage, a refined statistical analysis. We therefore decided for a compromise, and chose a slightly larger sample, still significant at the $\mathrm{p}<$ 0.10 level (orange area), in order to see whether the genes in that category pointed at particular functions non randomly, which would indicate that they are indeed significant. This approach is meant to propose genes as candidates for further study of pathogenicity, and a small number of false positives should not hinder further research in the domain, while false negatives would eliminate important candidates. Interestingly, the genes thus identified included phenol-soluble modulin (PSM) family peptides (SE0847), a Clp protease (SE0674), as well as genes involved in osmoprotection. Finally, 16 of the nonsynonymous significant genes were of unknown function. Careful analysis of these genes revealed however that they were likely to belong to functions important for pathogenicity, as indicated in Table 3. Several of those genes were counterparts of proteins generally conserved in Firmicutes (YvcD(SE0546), YheB(SE1527), YqfN(SE1247), YlaF(SE0806), YkyA(SE0790), YfmM(SE2128), YhfK(SE1745) and YqgE(SE1448)) and sometimes in larger Bacterial clades (SE1986). YvcD is a TPR-repeat protein, which might interact with nucleic acids; YkyA has a lipoprotein signal, and is similar to cell wall binding proteins; YqgE(SE1448) is similar to MultiDrug Efflux proteins and finally $\mathrm{YfmM}$ is likely to code for polyphosphate-AMP phosphotransferase. The very fact that they belong to this class of non synonymous SNPs makes them interesting candidates for further studies of pathogenicity.

Although the sequencing technique is quite accurate, the error frequency in a finished sequence is thought to be one error (frameshift or base substitution) in $10^{3}$ to $10^{5}$ bases [23]. To confirm the SNP sites identified in this study, we chose the top 13 pairs of mostly non-synonymous substitutions proteins and carried out PCR experiments on their cognate gene to validate the corresponding sequences. Sequences of the PCR products confirmed all the SNP sites we identified from the orthologs analysis.

Two main groups were observed in the cluster of 40 orthologous pairs made of proteins with mostly non-synonymous substitutions: surface proteins, which are likely under pressure to escape the host immune system, and other genes that should be considered in priority as important for pathogenicity. Two conserved hypothetical proteins (SE0265 and SE0378) predicted to localize in the extracellular medium and several transmembrane proteins, such as transporter family proteins, belonged to the first group. YkyA(SE0790), which contains a lipoprotein signal and a hydrolase domain, might also be recognized by the host immune defense. In the second group, several proteins involved in lipid metabolism, likely to be important for S. epidermidis multiplying on skin, apparently evolved faster in the pathogen. Genes encoding fosfomycin resistance protein FofB and beta-lactamase detected in this group also may have evolved fast to benefit bacteria trying to survive in their host. Genes involved in the formation of biofilms and osmoprotection were also found in this group. PSMs belong to the class of surfactant peptides with putative biofilm-inhibitory properties. Repression of expression of PSMs in the biofilm stage enables bacterial cells to adhere together and to evade the host immune system. Several gene products were involved in DNA recombination and repair (such as SE1170, SE1302 and SE1828) suggesting adaptation to some chemical stress. A gene (coding for methionine sulfoxide reductase, SE1042) involved in repair of Reactive Oxygen Species (ROS) damage, a process supposed to be significantly expressed upon infection [24], also suffered a high mutation rate. This prompted us to study the specific involvement of this process in the establishment of pathogenicity. Although the exact in vivo process of methionine oxygenation is not well established, it is supposed to be derived from ROS, in particular from reactions producing superoxide or $\mathrm{H}_{2} \mathrm{O}_{2}$ [25]. In an attempt to explore whether this prediction could be substantiated, both strains were challenged with increasing paraquat and $\mathrm{H}_{2} \mathrm{O}_{2}$ concentrations: interestingly, we observed that $S$. epidermidis ATCC12228 is indeed more sensitive to both paraquat [26] and $\mathrm{H}_{2} \mathrm{O}_{2}$ than S. epidermidis RP62A, as predicted (Figure 2 and 3, see Additional file 6 and Additional file 7).

Finally, genes are often grouped in operons, forming functionally consistent transcription units. If our hypothesis for the detection of genes important for pathogenesis is correct, then we should expect that a mutation bias would generally span entire operons when the corresponding genes participate to the process of pathogenicity. We found that genes making potential operons often displayed a collective increase in non-synonymous mutations (see Additional file 5). This is the case, for example, of potA (se0797) and potB (se0798), which code for the spermidine/putrescine $\mathrm{ABC}$ transporter.

\section{Discussion}

While an important topic for human health, pathogenicity is not the most prevalent development process for living organisms. Often, this particular way of life happens 


\section{A}

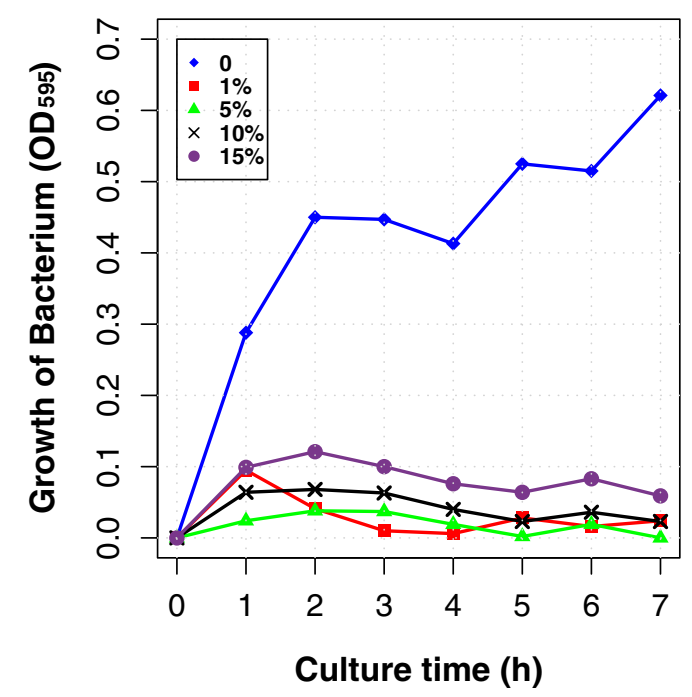

B

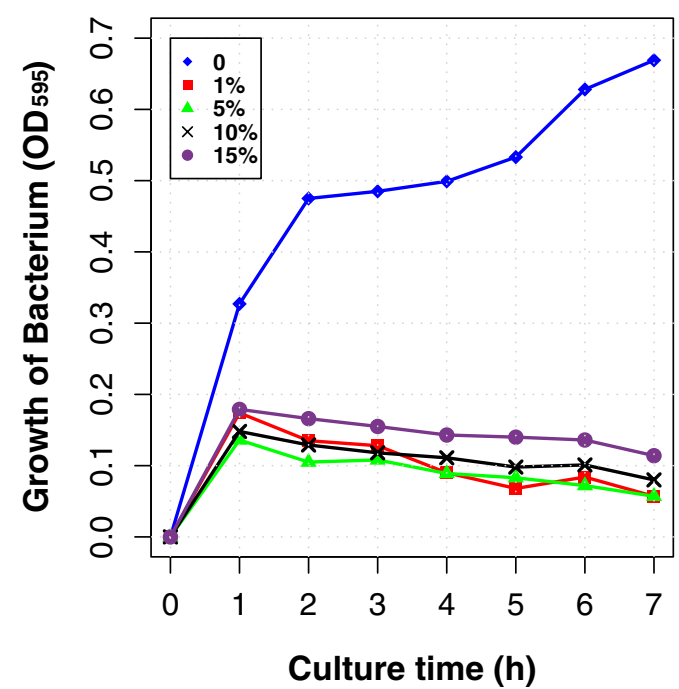

Figure 2

Sensitivity to $\mathrm{H}_{2} \mathrm{O}_{2}$ of the two S. epidermidis strains. Figure 2A displays the growth curve of S. epidermidis ATCCI 2228 and $2 \mathrm{~B}$, that of RP62A. The growth curves were plotted following the $\mathrm{OD}_{595}$ measured every one hour. Overnight bacterial cultures $\left(\mathrm{OD}_{595}\right.$ approximately I.2) were added to fresh TSB (Tryptone Soya Broth), and $\mathrm{H}_{2} \mathrm{O}_{2}$ at different concentrations ( 0 , $1 \%, 5 \%, 10 \%$ and $15 \%)$ were added at the same time. The culture density was monitored at $\mathrm{OD}_{595}$ followed every one hour.

as a new ecological niche (a possible host) is colonized by organisms that were previously indifferent or simple commensals. Previous work concluded, in general, that genome segments, often named pathogenicity islands, were the landmarks of pathogenic processes [27]. These islands are generally supposed to be the result of Horizontal Gene Transfer (HGT). HGT is a general process of gene acquisition by bacteria, where it may be associated to control of the background mutation level [28]. In Firmicutes, HGT is often the result of phage infection [29]. Bacteriophages can subsequently remain in the genome as functional (SPbeta in B. subtilis, for example) or more or less defective prophages (PBX, Skin, and similar elements). In the case of S. epidermidis, this type of analysis led Gill et al. to conclude that HGT was the major contribution to pathogenicity in the pathogenic strain they analyzed [12]. However, acquisition of virulence genes has repeatedly been found to be insufficient to trigger a pathogenic response. As a case in point, the cap operon, encoding the presumably virulence-associated polyglutamate capsule is present in both the commensal and pathogenic $S$. epidermidis strains [15]. The absence/presence of the ica operon that produces a biofilm exopolysaccharide was taken as the clearest genetic difference contributing to the different phenotype of two S. epidermidis strains [8,9]. However, the prevalence of icaADBC in commensal strains did not differ from that in invasive strains, indicating that other factors should been involved in pathogenesis [14]. All this indicates that important information about the development of pathogenic processes has been overlooked by the standard approaches focusing on HGT analysis. The small-scale variations which pepper the chromosomes of the commensal and pathogenic strains were not explored in detail. In order to gain further insight into the pathogenicity process, we studied the Single Nucleotide Polymorphisms (SNPs) between the orthologous gene pairs of the pathogenic and the commensal S. epidermidis strains of interest.

SNP analysis rests obviously on the quality of the genome sequences determination. The error frequency in a finished sequence has rarely been precisely measured. A common belief is that anywhere between one error in $10^{3}$ to $10^{5}$ bases, depending on the project. In order to evaluate the impact of sequencing errors on our analysis, approximately $23 \mathrm{~kb}$ of DNA sequence was re-sequenced after PCR amplification of 13 pairs of orthologs from both strains. 11 new SNP sites (5 and 6 in strains ATCC12228 and RP62A, respectively) were retrieved when the new sequences were compared to published genome sequences. If we take those new sites as sequencing errors, and not PCR errors or recent mutations, then the average error frequency is 4.8 errors in $10^{4}$ bases. This shows that, while we may have missed some interesting genes, our 


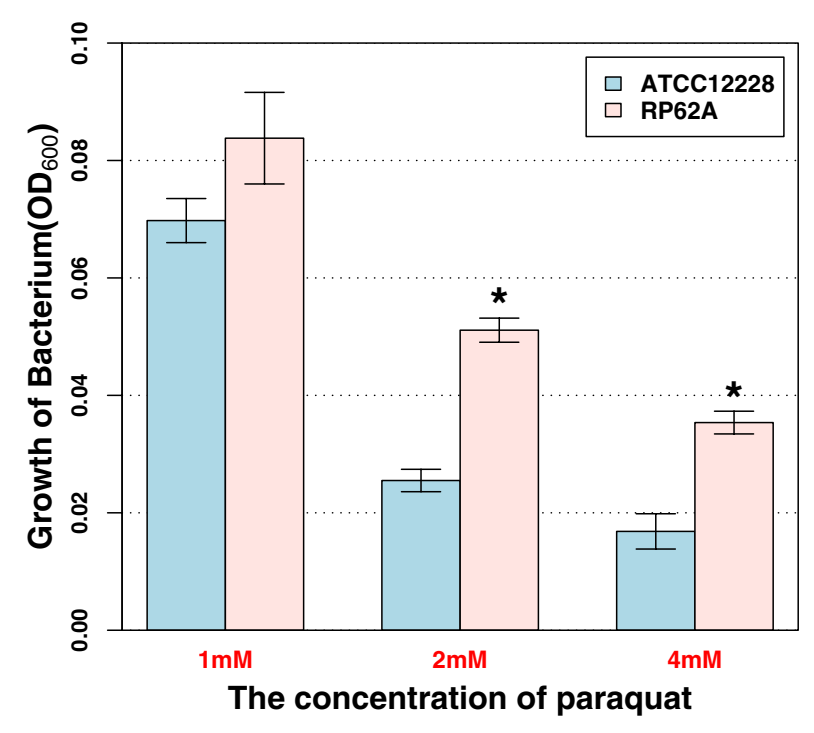

Figure 3

Sensitivity to paraquat of two $S$. epidermidis strains. The bacteria were cultured in MH broth (OXOID) with different concentration of paraquat (from I $\mathrm{mM}$ to $8 \mathrm{mM}$ ) and incubated at $35^{\circ} \mathrm{C}$ for $16-20 \mathrm{~h}$. The MIC was defined as the lowest concentration of paraquat giving complete inhibition of visible growth. The growth of bacteria incubated with lower concentration paraquat than MIC was measured by $\mathrm{OD}_{600}$. The bar graph represents the average of three independent experiments, with standard deviations denoted by the error bars. ${ }^{*}, P<0.01$.

approach is validated with the present available quality of genome sequences.

Analysis of the ratios of total non-synonymous SNPs vs total synonymous SNPs showed that N/S ratios for virulence factors and surface proteins differed significantly from that of total SNPs. While random mutations and evolutionary drift should not favor one type of mutation over the other one, genes related to pathogenic processes may have evolved recently due to positive selection to fit the pathogenic conditions [30]. Their evolution could be compared to that of genes belonging to translation, ribosomal structure and biogenesis (labeled according to the COG classification), which are submitted to purifying selection because of the general optimization of the translation process in the course of evolution. As expected, the latter were mostly submitted to synonymous mutations. This is remarkable and consistent with the interpretation of Drummond and colleagues who argued that highly expressed proteins evolve slowly because of the severe selective pressure on highly expressed proteins to avoid misfolding even when they are mistranslated [20]. This interpretation further supports the hypothesis that non synonymous mutations may be the landmark of proteins involved in pathogenic processes.

The percentages of identical vs. non-identical genes among the orthologs of those groups (virulence factors, surface proteins, translation-related genes and total genes) were also compared (see Additional file 9). Interestingly, the ratio of non-identical vs. identical genes coding for surface proteins differs significantly from that in translation-related genes and total genes, following the same trend as the ratio of non-synonymous vs. synonymous substitutions. This is not the case for virulence factors. The percentage of non-identical vs. identical genes reflected differences at the nucleic acids level, while the non-synonymous vs. synonymous differences emphasized the DNA mutations that result in a change protein sequences. Proteins are the ultimate functional entities in an organism, and this makes it reasonable to focus on the DNA mutations that change the coded amino acids, rather than silent mutations.

Of the 931 SNPs orthologous pairs identified in this study, a total of 40 genes showed a disproportionate distribution of $\mathrm{dN} / \mathrm{dS}$. Most are probably significant, as we consistently found that when present the imbalanced $\mathrm{dN} /$ dS distribution extended to genes common to an operon. That they may play important roles in pathogenic processes is suggested by the presence in the list of genes coding for proteins that are likely to influence pathogenicity, such as phenol-soluble modulin (PSM) family peptides (SE0847) or the Clp protease (SE0674). PSMs, a class of surfactant peptides with proinflammatory and putative biofilm-inhibitory properties, presumably represent key factors controlling the switch between the colonization and disseminative stages of the pathogen. Suppression of production of PSMs in the biofilm stage enables cells to stick together and to evade the host immune defense [31]. ATP-dependent Clp proteases are involved in regulation processes by proteolysis in many bacteria. They consist of a proteolytic subunit, $\mathrm{ClpP}$, which confers substrate specificity through association with ATPase subunits. Signature-tagged mutagenesis screening experiments have demonstrated that ATP-dependent proteases are key factors in bacterial adaptation to environmental stress, including ROS [32]. Little is known about clp gene regulation in pathogenic bacteria, despite the fact that many of these genes play important roles in virulence, such as ClpX in Staphylococcus aureus [33] and ClpB in Leishmania sp. [34]. It seems plausible that these bifunctional chaperones/proteases may also modulate the activity of other virulence factors [35] and it is therefore interesting to have observed that a member of this class is evolving fast in the S. epidermidis pair studied here. Genes involved in osmoprotection which may respond to high salt conditions in cell agglomerations on human skin, such as gbsA (glycine 
betaine aldehyde dehydrogenase) were also found among the non-synonymous significant genes.

Surface proteins play a fundamental role in the interaction between the bacterial cell and its environment. In the present case, several of the 40 non-synonymous genes, in particular some genes with unknown function, have features suggesting that they code for surface proteins under the pressure of escaping the host immune system. Interestingly, SE1527 and SE0790 are orthologous to surface proteins experimentally identified in one of Group A Streptococcus (GAS) strains (SPY0792 and SPY2018) [36]. It may also be significant that two ribosomal proteins, for which there is also some evidence of extracellular functions, were also found in their surface proteome. This makes RpsL (SE0309) and RplC (SE1824), the two ribosomal proteins which have more non-synonymous substitutions than expected in our analysis more interesting. RpsL, protein S12 of the small subunit of the ribosome is known to control accuracy of translation, and this might indeed play a role in pathogenicity [37]. Further work is required to investigate this aspect as we can expect that some of the genes identified are simply the result of random deviation from the average.

Yao et al have used microaray-based genome-wide comparison of clinical and commensal strains to identify putative virulence factors in S. epidermidis [38]. 39 genes were found to be more frequent among clinical strains than commensal strains. Interestingly, we find that after detailed comparison our 40 high-non-synonymous substitutions genes have no overlap with those 39 high-frequent genes in clinical strains. This is probably not unexpected, for the following reasons. Firstly, we looked for conserved genes rather than genes that would differ in a variety of strains, and our study involves only orthologous pairs of genes in the two stains, while Yao et al focused on those genes which showed disproportionate difference in distribution between clinical and commensal strains. Second, our 40 high non-synonymous substitution genes were identified because they carry SNPs, small differences which may not be detected by hybridization with the oligonucleotides of the microarray.

Under laboratory growth conditions, with plenty of metabolites available, bacterial gene expression is typically dominated by the highly expressed genes involved in transcription, protein biosynthesis, maturation and folding. In contrast, as shown for example in a study by Rollenhagen and Bumann, most of the genes highly expressed in Salmonella enterica cells recovered from the caecum differ considerably from those highly expressed in bacteria located in the spleen [39]. The overall functional profile of highly expressed genes suggests a marked shift in transcriptional activity upon change in growth environ- ment. In this respect, some pathogenicity related genes are likely to be highly expressed during infection to fight against the host. Interestingly, we found that one of the non-synonymous significant genes of the present study, gene se2269 encoding dihydropteroate synthase and recovered as a virulence-related antigen of a Gram-positive fish pathogen [40], was significantly more expressed in RP62A than in ATCC12228 (unpublished data).

Since pathogenicity is a recent form of lifestyle for an organism in evolution, we expected that genes involved in the process would show a significant mutation pattern [41]. Furthermore, we could expect that many of the corresponding mutations would not yet be adapted to the "self" of the bacteria, as they would result from non-synonymous mutations in codons, leading to alteration of the polypeptide sequence of the corresponding genes. Our observations substantiated this hypothesis, showing a significant number of genes, often grouped in operons, affected by non-synonymous mutations. In parallel - and this can be seen as an internal validation of our hypothesis - we observed that genes of the translation machinery, expected to be expressed at a high level (and hence under a "mutator" pressure) were indeed mutated, but that the corresponding mutations were usually of the synonymous class. This is easily accounted for by the enormous selection pressure operating on the translation machinery (made of proteins interacting with each other and with highly conserved RNAs), which cannot easily accommodate mutations resulting in alteration of the corresponding proteins. Even if important for pathogenicity, these genes would become invisible because the functional constraints would result in a purifying selection process. However not all genes can be equally submitted to purifying selection and our approach reveals several genes of the general cell machinery, as possibly important for pathogenicity. As a case in point, we found one gene (se1042) coding for peptide methionine sulfoxide reductase, involved in protection against ROS, which could probably be such an important candidate for adaptation to pathogenicity $[24,25]$. As the clpB gene found in our non-synonymous set also may point to adaptation to ROS, we explored the differential resistance to $\mathrm{H}_{2} \mathrm{O}_{2}$ and paraquat of the environmental species as compared to the pathogen (Figure 2 and 3, see Additional file 6 and file 7) and our experiments substantiated the interest of our approach to identify specific pathways of evolution to pathogenicity. In the same way a (presumably divalent) cation transporter SE0773 might be involved in the scavenging of divalent metals such as magnesium, manganese, iron or cobalt during infection. Gene se2128 might code for polyphosphate-AMP phosphotransferase, an observation that would support interest for the poorly explored role of polyphosphate in cells and its possible involvement in pathogenicity [42]. Others are involved in biofilm forma- 
tion, an important contribution to virulence in several pathogenic bacteria [43], as they considerably limit the success of both antibiotic treatment and the human immune defense. Gene expression profiling of the S. epidermidis biofilm was analyzed and some significant metabolic shift was found between the planktonic and the biofilm modes of growth [31].

\section{Conclusion}

In this paper, a SNPs comparative approach was developed to identify conserved genes possibly involved in pathogenicity by measuring their selective pressure in the gene pairs of non-infection associated $S$. epidermidis strain ATCC12228 and biofilm-forming strain RP62A. Our approach identified new genes that may be involved in pathogenesis, including some genes with unknown function. These results may provide fresh insight into discovering the genes that determine the success of $S$. epidermidis as an opportunistic pathogen. Complementing the previous methodologies which mainly focused on horizontal gene transfer, extensive SNPs investigations on more pathogens will facilitate our understanding of the path from commensalism to pathogenicity, a crucial prerequisite for designing therapeutic interventions directed to control pathogen infections.

\section{Methods}

\section{Orthologous genes identification}

For the identification of orthologous genes, all predicted CDSs from the S. epidermidis RP62A and ATCC12228 genomes were searched against each other locally using BLASTP [44]. Those genes that matched a non-self genomic sequence at $P$ value of $<=10^{-5}$, and identity $>=$ $35 \%$, matching at least $75 \%$ of the length of both query and subject sequences were considered homologous (non strain-specific genes). Of these homologous pairs, the bidirectional-best match was defined as an orthologous group. Identical nucleic acid sequence pairs, SNPs and insertion/deletions of the genes' pairs of orthologs between the two $S$. epidermidis genomes were identified using BLASTN.

\section{SNPs analysis}

The Nei-Gojobori (NG) method [45] was applied for estimating dS and $\mathrm{dN}$ of the SNPs pairs of S. epidermidis strains with minor modifications. The ratio of transition to transversion changes $(\mathrm{R})$ was obtained by counting the total numbers of transitions to transversions that were observed in the entire set of orthologous pairs of the two species and adjusted using Kimura's 2-parameters method [46]. The adjusted $\mathrm{R}$ computed from our data was 2.330294 and used in the following computation. We also applied the one-tail Z (or $t \infty$ ) test to conduct the statistical test of the positiveness of $\mathrm{dN}-\mathrm{dS}$. The estimated $\mathrm{dS}$ and $\mathrm{dN}$, standard error (S.E.), Z-scores and their corre- sponding p-values were presented in the additional file 5 . To explore the distribution of $\mathrm{dN} / \mathrm{dS}$, we applied the transformation $f(k)=\log (k+0.001)$ as described in other works[20,47].

\section{$\mathrm{H}_{2} \mathrm{O}_{2}$ and paraquat sensitivity analysis}

We grew the $\mathrm{S}$. epidermidis strains at different concentration of $\mathrm{H}_{2} \mathrm{O}_{2}$ and measured the growth curve of the strains by reading $\mathrm{OD}_{595}$ every one hour. Overnight bacterial cultures at $\left(\mathrm{OD}_{595}\right.$ approximately 1.2$)$ were added to fresh TSB(Tryptone Soya Broth) at a 1:100 dilution, and different concentrations of $\mathrm{H}_{2} \mathrm{O}_{2}(0,1 \%, 5 \%, 10 \%$ and $15 \%)$ were added at the same time. Cells were grown at $37^{\circ} \mathrm{C}$, agitated at, $220 \mathrm{rpm}$ and the absorbancy of the culture $\left(\mathrm{OD}_{595}\right)$ was followed every one hour.

MIC (minimal inhibitory concentration) assay of paraquat (sigma) by broth dilution was performed according to NCCLS (National Committee for Clinical Laboratory Standards) [48]. The bacterial inoculum was prepared using a 3-4 h broth culture of each isolates adjusted to a turbidity equivalent to a $0.5 \mathrm{McFarland}$ standard, diluted in CAMHB to achieve a final concentration of $5 \times 10^{5} \mathrm{CFU} / \mathrm{ml}$ in the test tube. Broth not containing an antimicrobial agent is inoculated as a control for organism viability, and E.coli ATCC 25922 was used as a test quality control strain. The bacteria were cultured in MH broth (OXOID) with different concentration of paraquat (from $1 \mathrm{mM}$ to $8 \mathrm{mM}$ ) and incubated at $35^{\circ} \mathrm{C}$ for $16-20 \mathrm{~h}$. The MIC was defined as the lowest concentration of paraquat giving complete inhibition of visible growth. The growth of bacteria incubated with lower concentration paraquat than MIC was measured by $\mathrm{OD}_{600}$.

\section{$P C R$ validation}

In order to get PCR fragments containing SNPs, we designed primers by Primer Premier 5.0. The primers sequences are listed in Additional file 8. We used Pfu DNA polymerase (Tiangen Biotechnology Co., Ltd) to ensure high-fidelity synthesis. Amplifications were carried out in a thermocycler (GeneAmp PCR system 9700) through the following temperature program: 1 cycle of $5 \mathrm{~min}$ at $94^{\circ} \mathrm{C}$; 30 cycles of $30 \mathrm{~s}$ at $94^{\circ} \mathrm{C}, 60 \mathrm{~s}$ at $55^{\circ} \mathrm{C}$, and $60 \mathrm{~s}$ at $72^{\circ} \mathrm{C}$; and finally 1 cycle at $72^{\circ} \mathrm{C}$ for $7 \mathrm{~min}$. The PCR products were purified by agarose gel DNA purification kit (TaKaRa Biotechnology Co., Ltd) and sequenced by Shanghai Invitrogen Biotechnology Co., Ltd.

\section{Abbreviations}

$\mathrm{dN}$, number of non-synonymous substitutions per site; dS, number of synonymous substitutions per site; CDS, Coding DNA sequence; SNPs, Single-nucleotide polymorphisms. 


\section{Authors' contributions}

WW performed the genome comparative analysis of the two strains and drafted the manuscript. ZWC contributed to conceive the study and draft the manuscript. YLZ contributed to $\mathrm{H}_{2} \mathrm{O}_{2}$ and paraquat sensitivity analysis and PCR experiments. XJW contributed to comparative analysis of the CDSs of both strains. GHD contributed to the statistical analysis of SNPs pairs. HX contributed to the identification of orthologs and PCR primer design. PLJ contributed to sequences analysis of PCR results. DQ contributed to conceive the study. AD conceived the rationale for the mutation analysis study and contributed to the writing of the manuscript. YXL contributed to conceive the study and revised the manuscript. All authors read and approved the final manuscript.

\section{Additional material}

\section{Additional File 1}

Distribution of insertions in either of the two sequenced Staphylococcus epidermidis genomes.

Click here for file

[http://www.biomedcentral.com/content/supplementary/14712164-7-112-S1.pdf]

\section{Additional File 2}

Distribution of orthologs of two Staphylococcus epidermidis strains. Click here for file

[http://www.biomedcentral.com/content/supplementary/14712164-7-112-S2.pdf]

\section{Additional File 3}

Ratios of non-synonymous vs synonymous of orthologs with SNPs pairs of Virulence factors.

Click here for file

[http://www.biomedcentral.com/content/supplementary/1471-

2164-7-112-S3.pdf]

\section{Additional File 4}

Ratios of non-synonymous $v$ s synonymous of orthologs with SNPs pairs of Surface proteins.

Click here for file

[http://www.biomedcentral.com/content/supplementary/1471-

2164-7-112-S4.pdf]

\section{Additional File 5}

Ratios of non-synonymous vs synonymous of orthologs with all SNPs pairs. Click here for file

[http://www.biomedcentral.com/content/supplementary/1471-

2164-7-112-S5.pdf]

\section{Additional File 6}

Comparison the sensitivity to $\mathrm{H}_{2} \mathrm{O}_{2}$ of both Staphylococcus epidermidis strains. Each bar represents the $\mathrm{OD}_{595}$ value of one strain at specific time and concentration of $\mathrm{H}_{2} \mathrm{O}_{2}$.

Click here for file

[http://www.biomedcentral.com/content/supplementary/1471-

2164-7-112-S6.pdf]

\section{Additional File 7}

Comparison the sensitivity to $\mathrm{H}_{2} \mathrm{O}_{2}(5 \%)$ of both Staphylococcus epidermidis strains. Red bars represent the $\mathrm{OD}_{595}$ value of $\mathrm{S}$. epidermidis RP62A at different time and blue bars represent $\mathrm{S}$. epidermidis ATCC12228.

Click here for file

[http://www.biomedcentral.com/content/supplementary/14712164-7-112-S7.pdf]

\section{Additional File 9}

Comparison of identical and non-identical distribution of different functional groups of orthologous genes.

Click here for file

[http://www.biomedcentral.com/content/supplementary/1471-

2164-7-112-S9.pdf]

\section{Additional File 8}

Primers used in the PCR experiments.

Click here for file

[http://www.biomedcentral.com/content/supplementary/14712164-7-112-S8.pdf]

\section{Acknowledgements}

We thank Zhongming Zhao for comments on the manuscript and useful discussion. This work was supported by Key Program of Basic Research of Shanghai (No. 04QMXI450, 03XDI4018, 02DJ 14002), the $863 \mathrm{Hi}-\mathrm{Tech}$ Program of China (No. 2003AA23 I0II, 2004BA7IIA2I, 2004AA223080) and the State Key Program of Basic Research of China (No. 2003CB7/5900, 2004CB5 I8606, 2002CB5 I2803). AD acknowledges the Blastsets program for support.

\section{References}

I. von Eiff C, Peters G, Heilmann C: Pathogenesis of infections due to coagulase-negative staphylococci. Lancet Infect Dis 2002, 2(I I):677-685

2. Mack D, Becker P, Chatterjee I, Dobinsky S, Knobloch JK, Peters G, Rohde $H$, Herrmann M: Mechanisms of biofilm formation in Staphylococcus epidermidis and Staphylococcus aureus: functional molecules, regulatory circuits, and adaptive responses. Int J Med Microbiol 2004, 294(2-3):203-2I2.

3. Peters G, Locci R, Pulverer G: Microbial colonization of prosthetic devices. II. Scanning electron microscopy of naturally infected intravenous catheters. Zentralbl Bakteriol Mikrobiol Hyg [B] 198I, I 73(5):293-299.

4. Christensen GD, Simpson WA, Bisno AL, Beachey EH: Adherence of slime-producing strains of Staphylococcus epidermidis to smooth surfaces. Infect Immun 1982, 37(I):318-326.

5. Gotz F: Staphylococcus and biofilms. Mol Microbiol 2002, 43(6): | $367-1378$.

6. Heilmann C, Gerke C, Perdreau-Remington F, Gotz F: Characterization of Tn9 17 insertion mutants of Staphylococcus epidermidis affected in biofilm formation. Infect Immun 1996, 64(I):277-282.

7. Heilmann C, Schweitzer O, Gerke C, Vanittanakom N, Mack D, Gotz F: Molecular basis of intercellular adhesion in the biofilmforming Staphylococcus epidermidis. Mol Microbiol 1996, 20(5): 1083-109|.

8. Galdbart JO, Allignet J, Tung HS, Ryden C, El Solh N: Screening for Staphylococcus epidermidis markers discriminating between skin-flora strains and those responsible for infections of joint prostheses. J Infect Dis 2000, I 82(I):35I-355.

9. Li H, Xu L, Wang J, Wen Y, Vuong C, Otto M, Gao Q: Conversion of Staphylococcus epidermidis strains from commensal to invasive by expression of the ica locus encoding production 
of biofilm exopolysaccharide. Infect Immun 2005, 73(5):3188-319|.

10. Vuong $C$, Otto $M$ : Staphylococcus epidermidis infections. Microbes Infect 2002, 4(4):48I-489.

II. Zhang YQ, Ren SX, Li HL, Wang YX, Fu G, Yang J, Qin ZQ, Miao YG, Wang WY, Chen RS, Shen Y, Chen Z, Yuan ZH, Zhao GP, Qu D, Danchin A, Wen YM: Genome-based analysis of virulence genes in a non-biofilm-forming Staphylococcus epidermidis strain (ATCC I 2228). Mol Microbiol 2003, 49(6): I577-I 593.

12. Gill SR, Fouts DE, Archer GL, Mongodin EF, Deboy RT, Ravel J, Paulsen IT, Kolonay JF, Brinkac L, Beanan M, Dodson RJ, Daugherty SC, Madupu R, Angiuoli SV, Durkin AS, Haft DH, Vamathevan J, Khouri H, Utterback T, Lee C, Dimitrov G, Jiang L, Qin H, Weidman J, Tran K, Kang K, Hance IR, Nelson KE, Fraser CM: Insights on evolution of virulence and resistance from the complete genome analysis of an early methicillin-resistant Staphylococcus aureus strain and a biofilm-producing methicillin-resistant Staphylococcus epidermidis strain. I Bacteriol 2005, I 87(7):2426-2438.

13. Glass JI, Belanger AE, Robertson GT: Streptococcus pneumoniae as a genomics platform for broad-spectrum antibiotic discovery. Curr Opin Microbiol 2002, 5(3):338-342.

14. Rohde H, Kalitzky M, Kroger N, Scherpe S, Horstkotte MA, Knobloch JK, Zander AR, Mack D: Detection of virulence-associated genes not useful for discriminating between invasive and commensal Staphylococcus epidermidis strains from a bone marrow transplant unit. J Clin Microbiol 2004, 42( I 2):56|4-56I9.

15. Kocianova S, Vuong C, Yao Y, Voyich JM, Fischer ER, DeLeo FR, Otto $M$ : Key role of poly-gamma-DL-glutamic acid in immune evasion and virulence of Staphylococcus epidermidis. J Clin Invest 2005, I I 5(3):688-694.

16. Herd $M$, Kocks $C$ : Gene fragments distinguishing an epidemicassociated strain from a virulent prototype strain of Listeria monocytogenes belong to a distinct functional subset of genes and partially cross-hybridize with other Listeria species. Infect Immun 200I, 69(6):3972-3979.

17. Stabler RA, Marsden GL, Witney AA, Li Y, Bentley SD, Tang CM, Hinds J: Identification of pathogen-specific genes through microarray analysis of pathogenic and commensal Neisseria species. Microbiology 2005, I 5 I (Pt 9):2907-2922.

18. Doumith $M$, Cazalet $C$, Simoes N, Frangeul L, Jacquet $C$, Kunst F, Martin $\mathrm{P}$, Cossart $\mathrm{P}$, Glaser $\mathrm{P}$, Buchrieser $\mathrm{C}$ : New aspects regarding evolution and virulence of Listeria monocytogenes revealed by comparative genomics and DNA arrays. Infect Immun 2004, 72(2): I072-1083.

19. Hommais F, Pereira S, Acquaviva C, Escobar-Paramo P, Denamur E: Single-nucleotide polymorphism phylotyping of Escherichia coli. Appl Environ Microbiol 2005, 7 I (8):4784-4792.

20. Drummond DA, Bloom JD, Adami C, Wilke CO, Arnold FH: Why highly expressed proteins evolve slowly. Proc Natl Acad Sci U S A 2005, I02(40): | $4338-14343$.

21. Jansen R, Gerstein M: Analysis of the yeast transcriptome with structural and functional categories: characterizing highly expressed proteins. Nucleic Acids Res 2000, 28(6): | $48 \mid$ I- 1488.

22. Dorus S, Vallender EJ, Evans PD, Anderson JR, Gilbert SL, Mahowald M, Wyckoff G], Malcom CM, Lahn BT: Accelerated evolution of nervous system genes in the origin of Homo sapiens. Cell 2004, I I (7): 1027-1040.

23. Weinstock GM: Genomics and bacterial pathogenesis. Emerg Infect Dis 2000, 6(5):496-504.

24. Weissbach H, Resnick L, Brot N: Methionine sulfoxide reductases: history and cellular role in protecting against oxidative damage. Biochim Biophys Acta 2005, 1703(2):203-2। 2.

25. Ezraty B, Aussel L, Barras F: Methionine sulfoxide reductases in prokaryotes. Biochim Biophys Acta 2005, I 703(2):22I-229.

26. Wen ZT, Suntharaligham P, Cvitkovitch DG, Burne RA: Trigger factor in Streptococcus mutans is involved in stress tolerance, competence development, and biofilm formation. Infect Immun 2005, 73(I):219-225.

27. Groisman EA, Casadesus J: The origin and evolution of human pathogens. Mol Microbiol 2005, 56(I): I-7.

28. Medigue C, Rouxel T, Vigier P, Henaut A, Danchin A: Evidence for horizontal gene transfer in Escherichia coli speciation. J Mol Biol I 99I, 222(4):85I-856.

29. Kunst F, Ogasawara N, Moszer I, Albertini AM, Alloni G, Azevedo V, Bertero MG, Bessieres P, Bolotin A, Borchert S, Borriss R, Boursier
L, Brans A, Braun M, Brignell SC, Bron S, Brouillet S, Bruschi CV, Caldwell B, Capuano V, Carter NM, Choi SK, Codani JJ, Connerton IF, Danchin A: The complete genome sequence of the grampositive bacterium Bacillus subtilis. Nature 1997, 390(6657):249-256.

30. Rocha EP, Danchin A: An analysis of determinants of amino acids substitution rates in bacterial proteins. Mol Biol Evol 2004, 2 I (I): 108-II6.

31. Yao Y, Sturdevant DE, Otto M: Genomewide analysis of gene expression in Staphylococcus epidermidis biofilms: insights into the pathophysiology of S. epidermidis biofilms and the role of phenol-soluble modulins in formation of biofilms. Infect Dis 2005, I 9 I (2):289-298.

32. Robertson GT, $\mathrm{Ng}$ WL, Foley J, Gilmour R, Winkler ME: Global transcriptional analysis of clpP mutations of type 2 Streptococcus pneumoniae and their effects on physiology and virulence. J Bacteriol 2002, I84(13):3508-3520.

33. Mei JM, Nourbakhsh F, Ford CW, Holden DW: Identification of Staphylococcus aureus virulence genes in a murine model of bacteraemia using signature-tagged mutagenesis. Mol Microbiol 1997, 26(2):399-407.

34. Schirmer EC, Glover JR, Singer MA, Lindquist S: HSPI 00/Clp proteins: a common mechanism explains diverse functions. Trends Biochem Sci 1996, 2 I(8):289-296.

35. Bae T, Banger AK, Wallace A, Glass EM, Aslund F, Schneewind O, Missiakas DM: Staphylococcus aureus virulence genes identified by bursa aurealis mutagenesis and nematode killing. Proc Natl Acad Sci U S A 2004, I0 I(33): 12312-12317.

36. Rodriguez-Ortega MJ, Norais N, Bensi G, Liberatori S, Capo S, Mora M, Scarselli M, Doro F, Ferrari G, Garaguso I, Maggi T, Neumann A, Covre A, Telford JL, Grandi G: Characterization and identification of vaccine candidate proteins through analysis of the group A Streptococcus surface proteome. Nat Biotechnol 2006, 24(2): $191-197$

37. Bjorkman J, Samuelsson P, Andersson DI, Hughes D: Novel ribosomal mutations affecting translational accuracy, antibiotic resistance and virulence of Salmonella typhimurium. Mol Microbiol 1999, 3 I(I):53-58.

38. Yao Y, Sturdevant DE, Villaruz A, Xu L, Gao Q, Otto M: Factors characterizing Staphylococcus epidermidis invasiveness determined by comparative genomics. Infect Immun 2005, 73(3): $1856-1860$.

39. Rollenhagen C, Bumann D: Salmonella enterica highly expressed genes are disease specific. Infect Immun 2006, 74(3): $1649-1660$

40. Hirono I, Yamashita $\mathrm{H}$, Park $\mathrm{Cl}$, Yoshida T, Aoki T: Identification of genes in a KG - phenotype of Lactococcus garvieae, a fish pathogenic bacterium, whose proteins react with antiKG rabbit serum. Microb Pathog 1999, 27(6):407-4I7.

41. Wright BE: Stress-directed adaptive mutations and evolution. Mol Microbiol 2004, 52(3):643-650.

42. Brown MR, Kornberg A: Inorganic polyphosphate in the origin and survival of species. Proc Natl Acad Sci U S A 2004, I0I(46): I6085-I6087.

43. Costerton JW, Stewart PS, Greenberg EP: Bacterial biofilms: a common cause of persistent infections. Science 1999 , 284(54 | 8): | $3 \mid 8-1322$.

44. Altschul SF, Madden TL, Schaffer AA, Zhang J, Zhang Z, Miller W, Lipman DJ: Gapped BLAST and PSI-BLAST: a new generation of protein database search programs. Nucleic Acids Res 1997, 25(17):3389-3402.

45. Zhang J, Rosenberg HF, Nei M: Positive Darwinian selection after gene duplication in primate ribonuclease genes. Proc Natl Acad Sci U S A 1998, 95(7):3708-37। 3.

46. Kimura M: A simple method for estimating evolutionary rates of base substitutions through comparative studies of nucleotide sequences. J Mol Evol I980, I6(2): I II-I 20.

47. Wall DP, Hirsh AE, Fraser HB, Kumm J, Giaever G, Eisen MB, Feldman MW: Functional genomic analysis of the rates of protein evolution. Proc Natl Acad Sci U S A 2005, I 02(I 5):5483-5488.

48. National Committee For Clinical Laboratory Standards: Performance standards for antimicrobial susceptibility testing; fourteenth Informational supplement MI00-SI4. In NCCLS Villanova, PA, USA; 2004. 